\title{
Ovarian Endometriotic Cyst
}

National Cancer Institute

\section{Source}

National Cancer Institute. Ovarian Endometriotic Cyst. NCI Thesaurus. Code C4069.

A cystic form of endometriosis affecting the ovary. It may or may not be associated with endometriosis in other areas in the pelvis. 